\title{
Finding the Pion in the Chiral Random Matrix Vacuum
}

\author{
G. W. Carter and A. D. Jackson \\ The Niels Bohr Institute, Blegdamsvej 17 \\ DK-2100 Copenhagen $\varnothing$, Denmark
}

(August 11, 2018)

\begin{abstract}
The existence of a Goldstone boson is demonstrated in chiral random matrix theory. After determining the effective coupling and calculating the scalar and pseudoscalar propagators, a random phase approximation summation reveals the massless pion and massive sigma modes expected whenever chiral symmetry is spontaneously broken.
\end{abstract}

PACS: 11.30.Rd, 11.30.Qc, 12.38.Lg.

Increasing evidence supports the applicability of chiral random matrix theory $(\chi \mathrm{RMT})$ [1] for describing the spontaneous breaking of chiral symmetry in quantum chromodynamics (QCD). (For a review, see Ref. [2].) Using the Banks-Casher relation [3], the vacuum chiral condensate, $\Sigma$, can be related to the spectral density of the Dirac operator in the vicinity of zero eigenvalue. This model has been extended to describe chiral symmetry restoration, for both finite temperature and baryon chemical potential [4], in what amounts to a mean field treatment. Although these efforts have led to a schematic phase diagram for QCD as a function of $T, \mu$, and the current quark mass, $m$, other familiar vacuum phenomena associated with chiral symmetry breaking remain to be obtained. In this letter, we consider quark-antiquark correlation functions in order to recover the Goldstone mode. This is a ubiquitous feature associated with the spontaneous breaking of a continuous global symmetry which is expected whenever the symmetry group is of order greater than one. In $\chi \mathrm{RMT}, \mathrm{SU}_{L}\left(N_{f}\right) \times \mathrm{SU}_{R}\left(N_{f}\right)$ chiral symmetry is broken into $\mathrm{SU}_{V}\left(N_{f}\right)$ as is believed to occur in simplified QCD with two massless flavors. Along with the chiral condensate, $\langle\bar{\psi} \psi\rangle_{0}$, we should expect $\chi \mathrm{RMT}$ to provide us with massless pseudoscalar (i.e., the pion) and massive scalar (i.e., the sigma) excitations.

In order to extract this feature from a model of chiral symmetry breaking at the quark level, a resummation in the spirit of the random phase approximation (RPA) can be performed to construct the current-current correlation function in a given channel. This is shown schematically in Fig. 1. Evidently, this procedure requires two modeldependent quantities: an effective interaction between quarks and a correlator describing the propagation of a quark-antiquark pair in the gluonic background. If we denote the coupling as $g$ and the "bare" $q \bar{q}$ propagator as $\Pi_{0}$, the full or "dressed" correlation function, $\Pi$, can be written as an infinite sum. Each term in this sum is an alternating string of coupling constants and bare propagators as indicated in Fig. 1. This geometric series is readily written as:

$$
\Pi=\frac{g}{1-\Pi_{0} g} .
$$

In principle, both $g$ and $\Pi_{0}$ can depend on the momentum carried by the quark-antiquark pair in a model-dependent manner. We now turn to the particular case of $\chi \mathrm{RMT}$.

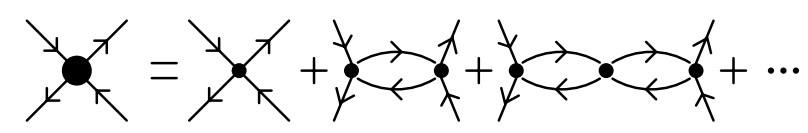

FIG. 1. The $q \bar{q}$ propagator in the random phase approximation.

To determine $\Pi$, we assume a factorization of the elements $g$ and $\Pi_{0}$, in which case its solution takes the RPAlike form of Eq. (1) and Fig. 1. The coupling constant is computed as the average strength of the four-quark interaction induced by the random matrix background. Next, $\Pi_{0}$ is schematically associated with the two-eigenvalue correlation function, calculable in $\chi \mathrm{RMT}$, and evaluated in the appropriate limits. This is the zero-dimensional analogue of a loop integral, in that it encodes the effects of the chiral condensate on a quark-antiquark pair.

The effective coupling, $g$, is easily determined by averaging over the random matrix elements in the partition function of $\chi \mathrm{RMT}$ in order to generate an effective quark interaction. This partition function can be written as

$$
\begin{aligned}
\mathcal{Z}=\int \mathcal{D} \psi^{\dagger} \mathcal{D} \psi \mathcal{D} W \exp \left\{\sum_{j=1}^{N_{f}} \psi_{j}^{\dagger}\left(\begin{array}{cc}
0 & i W \\
i W^{\dagger} & 0
\end{array}\right) \psi^{j}\right. \\
\left.-n \Sigma^{2} \operatorname{Tr}\left[W^{\dagger} W\right]\right\},
\end{aligned}
$$

where the $W$ are $n \times n$ complex matrices and where $\psi$ and $\psi^{\dagger}$ are row and column vectors with $2 n$ components representing two-component chiral spinors. Implicit in this form is the choice of a Gaussian unitary ensemble (GUE), appropriate for the $\mathrm{SU}(3)$ gauge group, with variance $\Sigma$. The choice of a Gaussian potential is not mandatory in chiral random matrix theory since the microscopic spectral correlators have been shown to be universal for a wide class of potentials [5.6].

The integral over $W$ in Eq. (2) can be performed to obtain 


$$
\begin{aligned}
\mathcal{Z}= & \int \mathcal{D} \psi^{\dagger} \mathcal{D} \psi \\
& \exp \left(-\sum_{i, j=1}^{N_{f}} \sum_{\alpha, \beta=1}^{n} \frac{1}{n \Sigma^{2}} \psi_{L i \alpha}^{\dagger} \psi_{R}^{i \beta} \psi_{R j \beta}^{\dagger} \psi_{L}^{j \alpha}\right),
\end{aligned}
$$

from which one immediately finds an induced four-quark interaction. After normalizing for $N$, the effective coupling strength is $g=\left(2 N_{f} \Sigma^{2}\right)^{-1}$. This coupling is constant, as is to be expected from the mean-field nature of random matrix theory.

The second ingredient, the bare current-current correlation function, has the form

$$
\begin{aligned}
\Pi_{0} \Gamma\left(q^{2}\right) & =\int d^{4} x \mathrm{e}^{-i q \cdot x}\langle\bar{\psi}(x) \Gamma \psi(x) \bar{\psi}(0) \Gamma \psi(0)\rangle \\
& =\int \frac{d^{4} p}{(2 \pi)^{4}} \operatorname{Tr}[i S(p) \Gamma i S(p-q) \Gamma] .
\end{aligned}
$$

Here, $S(q)$ is the fermion propagator, $\Gamma$ is one of the 16 Dirac basis matrices which generate the Clifford algebra, and $q$ is the momentum carried by the quark-antiquark pair. The two relevant correlators here are the identity matrix $\Gamma=\mathbf{1}$ and $\Gamma=\gamma_{5}$ appropriate for the scalar and pseudoscalar channels, respectively. We will be concerned only with results valid in the $q \rightarrow 0$ limit of zero momentum transfer.

Fortunately, the bare propagators of Eq. (4) are directly related to the function, $\rho\left(\lambda_{1}, \lambda_{2}\right)$, describing the correlation between two eigenvalues of the Dirac operator. This spectral correlator is accessible in $\chi \mathrm{RMT}$ and, moreover, known to be universal in the microscopic limit. The calculation of spectral correlators is usually technically involved, but it simplifies significantly in random matrix theory through the use of orthogonal polynomial techniques [7]. The desired spectral correlator is

$$
\rho\left(\lambda_{1}, \lambda_{2}\right)=\left\langle\frac{1}{N} \operatorname{Tr} \frac{1}{\lambda_{1}-H} \frac{1}{N} \operatorname{Tr} \frac{1}{\lambda_{2}-H}\right\rangle
$$

for eigenvalues $\lambda_{1}$ and $\lambda_{2}$ generated by averaging over $N \times N$ matrices $H$ with elements drawn from a random Gaussian distribution. For problems with chiral symmetry, $H$ has the form

$$
H=\left(\begin{array}{cc}
0 & i W \\
i W^{\dagger} & 0
\end{array}\right) .
$$

The scalar and pseudoscalar correlation functions of Eq. (4) can be constructed from this spectral correlator. We will be interested in taking limits $N \rightarrow \infty$ and $\Delta \equiv\left(\lambda_{1}-\lambda_{2}\right) \rightarrow 0$ simultaneously. This requires first fixing $\Delta \sim 1 / N$ and then taking $N \rightarrow \infty$. Since the effective coupling of Eq. (3) does not depend on the momentum transferred, we are interested ultimately in the limit in which $\lambda \rightarrow 0$. The limits cannot be interchanged, for their ordering is crucial in obtaining the physically relevant quantities from $\chi \mathrm{RMT}$ [8].
To extract the $\Delta$ dependence in the described limit we use the microscopic spectral correlation function,

$$
\rho_{M}(x, y)=\lim _{N \rightarrow \infty} \rho(x / N \Sigma, y / N \Sigma) .
$$

The microscopic limit is taken in order to retain information on the scale of the spacing between neighboring eigenvalues, the scale of $\delta \equiv \Delta N \Sigma$ which corresponds to the momentum $q$. At the same time the eigenvalues themselves are rescaled into microscopic variables $x=\lambda N \Sigma$ and the limit $\lambda \rightarrow 0$ is replaced by $x \gg 1$. Thus, we are considering two eigenvalues which are close on the macroscopic scale, but have a difference much greater than the average spacing between microscopic, unfolded eigenvalues.

In these limits the real parts of the traces of Eq. (5) vanish, being odd in $\lambda$, and we need only consider the contributions from the imaginary parts. These may be found by taking the discontinuities across the real axes in the limit of zero current masses $m, m^{\prime}$. For a single fermion species,

$$
\begin{aligned}
\lim _{q^{2} \rightarrow 0} \Pi\left(q^{2}\right) & =\left.\lim _{q^{2} \rightarrow 0} \lim _{m, m^{\prime} \rightarrow 0} \operatorname{Disc}\right|_{m=i \lambda, m^{\prime}=i \lambda^{\prime}} \Pi\left(q^{2} ; m, m^{\prime}\right) \\
& =\pi^{2} \lim _{\delta \rightarrow 0} \lim _{x \gg 1} \rho_{M}(x, x-\delta) .
\end{aligned}
$$

Knowing that $\gamma_{5}$ reflects left-handed quark eigenfunctions and leaves right-handed eigenfunctions unchanged, we find the bare scalar and pseudoscalar correlation functions in the appropriate limits:

$$
\begin{gathered}
\lim _{q^{2} \rightarrow 0} \Pi_{0}{ }^{\mathrm{S}}\left(q^{2}\right)=2 N_{f} \pi^{2} \lim _{\delta \rightarrow 0} \lim _{x \gg 1} \rho_{M}(x, x-\delta), \\
\lim _{q^{2} \rightarrow 0} \Pi_{0}{ }^{\mathrm{PS}}\left(q^{2}\right)=-2 N_{f} \pi^{2} \lim _{\delta \rightarrow 0} \lim _{x \gg 1} \rho_{M}(x, x-\delta) .
\end{gathered}
$$

The exact expression for the microscopic two point correlation function, $\rho_{M}(x, y)$, was found in Ref. [9] for chiral ensembles. The connected part is

$$
\begin{gathered}
\rho_{M}(x, y)=\frac{\bar{x} \bar{y}}{\left(x^{2}-y^{2}\right)^{2}}\left[x J_{N_{f}}(\bar{x}) J_{N_{f}-1}(\bar{y})\right. \\
\left.-y J_{N_{f}-1}(\bar{x}) J_{N_{f}}(\bar{y})\right]^{2},
\end{gathered}
$$

where $\bar{x}=x \pi \rho(0) / \Sigma$, with $\rho(\lambda)$ being the continuum single eigenvalue spectrum.

Fixing $\delta$ as previously explained, the above equation (9) has the limit

$$
\lim _{x \gg 1} \rho_{M}(x, x-\delta)=\left(\frac{\sin \left[\delta \pi \rho(0) / \Sigma^{2}\right]}{\delta \pi / \Sigma^{2}}\right)^{2} .
$$

The distinction between the scalar and pseudoscalar correlators naturally lies in the sign difference between the two. The argument $\delta$ is a small but finite number which can be associated with the Minkowski momentum, $q$, carried by the quark-antiquark pair. Expanding 
Eq. (10) in small $\delta$ and inserting this into Eq. (8), the quark correlation functions are found to be

$$
\Pi_{0} \mathrm{~S}, \mathrm{PS}\left(q^{2}\right)= \pm 2 N_{f}\left(\frac{\pi^{4} \rho(0)^{4}}{3 \Sigma^{4}} q^{2}-\pi^{2} \rho(0)^{2}\right)+\mathcal{O}\left(q^{4}\right)
$$

valid in the limit of small $q$.

Now, we need only perform the sum indicated in Fig. 1. The full correlation function, $\Pi^{\Gamma}\left(q^{2}\right)$, is summed as Eq. (11):

$$
\Pi^{\Gamma}\left(q^{2}\right)=\frac{1}{2 N_{f} \Sigma^{2}-\Pi_{0}^{\Gamma}\left(q^{2}\right)} .
$$

Inserting the solutions for each channel, Eqs. (11), we find

$$
\Pi^{S}\left(q^{2}\right)=-\frac{C}{q^{2}-2 N_{f} C\left[\pi^{2} \rho(0)^{2}+\Sigma^{2}\right]},
$$

for the scalar channel, where $C=3 \Sigma^{4} /\left[2 N_{f} \pi^{4} \rho(0)^{4}\right]$. For the pseudoscalar channel we obtain

$$
\Pi^{P S}\left(q^{2}\right)=\frac{C}{q^{2}-2 N_{f} C\left[\pi^{2} \rho(0)^{2}-\Sigma^{2}\right]} .
$$

The scalar propagator has a pole at finite $q^{2}$. This result is consistent with our expectation of a massive excitation. (This is simply the scalar mesonic mode, usually denoted $\sigma$.) On the other hand, we expect that pseudoscalar excitations should be identified with the pion and, hence, should be massless. Indeed, by virtue of the Banks-Casher relation,

$$
\Sigma=\pi \rho(0),
$$

we see that the pseudoscalar propagator assumes the form

$$
\Pi^{P S}\left(q^{2}\right)=\frac{C}{q^{2}},
$$

exhibiting the pole at $q^{2}=0$ indicative of a Goldstone boson. This cancellation is precisely analogous to that which occurs when a gap equation, arising in an effective field approach (e.g., the Nambu-Jona-Lasinio or instanton vacuum models), conspires with the $q$-independent part of the pseudoscalar correlator to leave a massless mode. Here, $\chi \mathrm{RMT}$ exhibits a corresponding content in that a relation between the chiral condensate and the single-quark energy spectrum is vital for recovering the pion as a massless excitation. Furthermore, the collapse of support at zero eigenvalue with the restoration of chiral symmetry [8] mimics the gap equation's loss of validity when the condensate vanishes.

Finally, we note that the scalar propagator of Eq. (13) implies an effective mass of $M^{2}=6 \Sigma^{2}$. Because of the ambiguity of scale in our association between eigenvalues and momenta this cannot be taken as a precise numerical prediction. However, it is clear that the mass scales with the strength of the condensate, $\Sigma$, as is common in effective field models.

The present results were obtained with Gaussian distributed random matrix elements. However, the demonstration of massless pseudoscalar and massive scalar excitations relied only on properties of the microscopic spectral correlators which are known to be universal. Thus, we expect that the present qualitative results will apply to a greatly extended class of potentials.

By following the path of an RPA resummation and utilizing exact results from random matrix theory in the thermodynamic limit, we have demonstrated that evidence of spontaneously broken chiral symmetry based on the Banks-Casher relation is confirmed by the existence of Goldstone modes with mesonic quantum numbers. This result indicates that $\chi \mathrm{RMT}$ inherently contains phenomena beyond the mere appearance of a chiral condensate. It also suggests that the pattern of spontaneous symmetry breaking found in $\chi \mathrm{RMT}$ is the same as that occurring in dynamical field theoretic approaches. Finally, the underlying connection between the vacuum condensate and the single-quark spectrum is further seen to be a feature which chiral random matrix theory shares with other descriptions of chiral symmetry breaking.

We thank W. Weise for a particularly useful discussion, B. Vanderheyden for numerous conversations, A. Polleri for a discussion of the NJL model, and K. Splittorff for a critical reading of this manuscript.

[1] E. Shuryak and J.J.M. Verbaarschot, Nucl. Phys. A560, 306 (1993).

[2] J.J.M. Verbaarschot, The Infrared Limit of the QCD Dirac Spectrum and Applications of Chiral Random Matrix Theory to $Q C D$, hep-ph/9902394

[3] T. Banks and A. Casher, Nucl. Phys. B169, 103 (1980).

[4] M.A. Halasz, A.D. Jackson, R.E. Shrock, M.A. Stephanov and J.J.M. Verbaarschot, Phys. Rev. D 58, 096007 (1998).

[5] A.D. Jackson, M.K. Şener and J.J.M. Verbaarschot, Nucl. Phys. B506, 612 (1997).

[6] G. Akemann, P.H. Damgaard, U. Magnea and S.M. Nishigaki, Nucl. Phys. B519, 682 (1998).

[7] M. Metha, Random Matrices (Academic Press, San Diego, 1991).

[8] A.D. Jackson and J.J.M. Verbaarschot, Phys. Rev. D 53, 7223 (1996).

[9] J.J.M. Verbaarschot and I. Zahed, Phys. Rev. Lett. 70, 3852 (1993). 